\title{
Distributed Model Predictive Control for Energy Management in a Network of Microgrids using the Dual Decomposition Method
}

\author{
Matteo Razzanelli ${ }^{1}$ | Emanuele Crisostomi ${ }^{2}$ | Lucia Pallottino ${ }^{1,3}$ | Gabriele Pannocchia*4
}

\author{
${ }^{1}$ Dipartimento di Ingegneria \\ dell'Informazione, University of Pisa, L.go \\ L. Lazzarino, 56122, Pisa, Italy \\ ${ }^{2}$ Dipartimento di Ingegneria dell'Energia, \\ dei Sistemi, del Territorio e delle \\ Costruzioni, University of Pisa, L.go L. \\ Lazzarino, 56122, Pisa, Italy \\ ${ }^{3}$ Research Center "E. Piaggio", University of \\ Pisa, L.go L. Lazzarino, 56122, Pisa, Italy \\ ${ }^{4}$ Dipartimento di Ingegneria Civile e \\ Industriale, University of Pisa, L.go L. \\ Lazzarino, 56122, Pisa, Italy \\ Correspondence \\ *Corresponding author. Email: \\ gabriele.pannocchia@unipi.it
}

\begin{abstract}
Summary
This paper deals with the application of Model Predictive Control (MPC) to optimize power flows in a network of interconnected MicroGrids (MGs). More specifically, a Distributed MPC (DMPC) approach is used to compute for each MG how much active power should be exchanged with other MGs and with the outer power grid. Due to the presence of coupled variables, the DMPC approach must be used in a suitable way to guarantee the feasibility of the consensus procedure among the MGs. For this purpose, we adopt a tailored dual decomposition method that allows us to reach a feasible solution while guaranteeing the privacy of single MGs (i.e., without having to share private information like the amount of generated energy or locally consumed energy). Simulation results demonstrate the features of the proposed cooperative control strategy and the obtained benefits with respect to other classical centralized control methods.
\end{abstract}

KEYWORDS:

Microgrids, model predictive control, distributed systems, dual decomposition

\section{1 | INTRODUCTION}

\section{1 | Motivation}

Electric MicroGrids (MGs) have been the subject of intense research during the last decade. While the initial interest in MGs was mainly motivated by the possibility of using them to supply energy in remote regions, recent advances in power grid technologies and the increasing penetration of distributed energy resources are also considering the possibility of splitting the already existing power grids into semi-independent MGs. Among others, expected advantages of this choice include a better grid resiliency, a simpler control hierarchy, a more flexible resource allocation, and an increased robustness against unexpected failures in the power grid $^{1,2,3}$.

Roughly speaking, a MG is defined as a smart power system of small size that includes (i) conventional power generators and renewable-based generators; (ii) loads, and (iii) energy storage devices (e.g., most notably batteries, but in general also thermal storage devices and possibly plug-in electric vehicles), grouped together within a limited geographic region ${ }^{4,5}$. However, the main property of a MG is its ability to both operate connected to the main power grid, interconnected to other MGs, or also disconnected from other power grids (i.e., in island mode).

The Energy Management System (EMS) represents the core of the MG, as it is responsible of taking the most convenient choices for the MGs. Among others, the EMS chooses the optimal mode of the MG (e.g., connected or disconnected from the outer grid), it optimizes the power flows within the MG (e.g., deciding which power generators should be used to provide the required energy), and it tries to maximize the revenues of the MG by selling the exceed energy to other MGs, or to the outer 
grid. In this context, the presence of storage devices adds a further degree of flexibility, as storage devices may either behave as conventional loads (i.e., when they are charged) or may be used to provide energy (i.e., when they are discharged).

The first papers on this topic ${ }^{6,7}$ only addressed the problem of optimizing the decisions of single MGs, neglecting the existing interactions among different MGs. More recently, there is an increasing interest in investigating how the interactions among MGs may improve the operation of single MGs ${ }^{1}$, and in general affect the stability of the power grid $^{8}$. Due to the difficulty of predicting a future behavior of uncertain variables, most notably, the amount of energy that will be generated from renewable sources, most optimization strategies that have been proposed in the literature either focus on instantaneous, or greedy, optimization ${ }^{8}$ neglecting what will happen in a future horizon, or have assumed that future forecasts of uncertain variables may be regarded as perfectly accurate ${ }^{9}$.

In this paper, we explicitly take into account a future time horizon, and we do so by adopting a popular receding horizon control strategy like Model Predictive Control (MPC). MPC is a class of advanced control techniques, widely used especially in the context of process industries, mainly due to its ability to ensure closed-loop stability and optimality under constraint satisfaction. Also, this strategy is known to be robust also for many classes of multivariable linear and nonlinear systems ${ }^{10,11,12}$.

The global performance of a large-scale power system consisting of many interconnected microgrids generally depends on the amount of information that is exchanged among the MGs. One possibility is to assume that all MGs are willing to exchange their local information with all the other MGs to cooperatively achieve a common goal. Then, a single MPC algorithm shared among all the interconnected MGs can be used to achieve the optimal performance ${ }^{13,14,15,16}$. However, if we assume that MGs have the personal interest of maximizing their own revenues (e.g., by selling energy to other MGs), and that they may be owned by different energy providers, then it is more realistic to consider that MGs will be actually reluctant to reveal local information, e.g., how much power is generated, or the state of charge of their storage devices. In addition to this, decentralized/distributed solutions are known to be more scalable than centralized solutions, especially when the overall number of subsystems becomes large. Even if this limitation does not hold, it is often the case that organizational reasons impose the use of smaller local controllers, which are easier to maintain and operate ${ }^{17,18}$. Moreover, a distributed solution is also prone to possible faults or deliberated attacks since in that case they guarantee a controlled behaviour of at least a part of the network. For the aforementioned reasons, we take a distributed approach in this paper as well.

\section{2 | Related works}

A distributed control architecture to solve the power scheduling approach, so that the aggregate demand equals the supply, is considered in ${ }^{19}$. Similarly, a distributed peer-to-peer multi-agent framework is proposed for solving the power sharing problem in MGs with inverter-interfaced distributed energy resources ${ }^{20}$. A recent paper reported approaches such as ADMM, and distributed population dynamics ${ }^{21}$, that could also solve this problem. Recently, a cooperative MPC-based approach for energy management in a network of MGs was also formulated in order to achieve a common goal ${ }^{22}$. Also, less conventional ways of implementing cooperative control actions in an MPC framework can be found in literature ${ }^{23}$.

More in detail, a recent work presented a decentralized MPC for tracking formulation, and it proposed an algorithm based on the dual decomposition algorithm ${ }^{24}$. For this purpose, a slack variable is added to the objective function to penalize deviations from a perfect balancing between generated and consumed power. On the other hand, in our case, we consider the power balance as a hard constraint, and we rather maximize the revenues of the MGs once the constraint is satisfied.

A dual decomposition method is presented by Zhang et al. ${ }^{19}$ as well, but the obtained solution is implemented in open-loop, i.e. the optimization problem is not solved at each time as in MPC framework.

On the other hand, a specific paper greatly inspired our work here ${ }^{25}$. In the aforementioned work, a cooperative MPC algorithm with a global objective function is defined. While most of the assumptions and test cases in our paper are taken from the previous mentioned paper, the main difference is that we formulate the optimization problem in a distributed way, while a centralized approach have been adopted in the above one ${ }^{25}$. In our opinion, a distributed solution has many advantages, and most notably in this context it allows MGs not to reveal sensitive information. An interesting approach to avoid a safe information exchange is proposed in ${ }^{26}$.

In the recent literature, a distributed solution is also investigated ${ }^{20}$ as well, even though a PI control is used instead of MPC. Finally, a distributed cooperative MPC approach has been already proposed ${ }^{22}$. However, this latter solution ${ }^{22}$ assumes the presence of an aggregator that collects information of single MGs to centrally solve the optimization problem. On the other hand, our distributed MPC solution can be applied by using a similar aggregator, but also without it, in a completely distributed 
way. The Distribution System Operator (DSO) as well as a third-role party may be interested in playing the role of aggregator. However it is not in the scope of this paper to know who might play the role of the aggregator.

\section{3 | Original contribution}

As from the discussion in Section 1.2, although MPC methods have been already used to optimally schedule power flows in a network of MGs, some aspects have not been completely tackled yet. For instance, how to implement this scenario without using an aggregator to handle the coupled variables (e.g., the power exchanged between two MGs). In this context, our paper proposes a way to do so without requiring the presence of an aggregator. In particular, we propose here a fully distributed MPC solution, e.g., without the presence of aggregators, which is a novel solution in this context. Such distributed solutions may be highly desirable in terms of low communication burden and safer information exchange. The rest of this paper is organized as follows. In Section 2, the MG system is described and a brief review of a centralized predictive formulation is given. A Distributed MPC scheme and the potential issues derived from this formulation for a power scheduling problem are formalized in Section 3. Then, the proposed distributed predictive energy management system to overcome these issues is presented in Section 3.2. Finally some simulation scenarios are described in Section 4 and conclusions are presented in Section 5.

\section{2 | A CENTRALIZED FORMULATION OF THE OPTIMAL POWER FLOW PROBLEM IN A NETWORK OF MGS}

\section{1 | Problem description}

MGs generally consist of Distributed Energy Resources (this may both include conventional and renewable-based DERs), storage devices and uncontrollable loads. MGs are connected at the level of the Distribution Network and may either exchange power among themselves or with the Distributed Network Operator (DNO), as exemplified in Figure 1 where the network of MGs is depicted together with the physical links for power exchange. Accordingly, the network of MGs is equivalent to a graph $\mathcal{G}=(\mathcal{V}, \mathcal{E})$, where the MGs correspond to the set of nodes $\mathcal{V}$ that communicate and exchange power among themselves by means of a set of edges $\mathcal{E}$. Here we consider that the edges both refer to the communication links and to the power exchange links, though this is not required in principle. The only required assumption is that both the communication and the power exchange graphs need to be connected ${ }^{27}$. The objective of each MG is to provide the required power to serve all the connected loads, while minimizing the operational costs for doing so. In this context, we shall use negative costs to represent (positive) profits. Under the assumption that each MG has a 24-hour ahead accurate hourly forecast of the power demand/generation imbalances ${ }^{25}$, the local controller of each MG can decide to exchange power with either another MG or with the DNO, on the basis of its convenience (e.g., on the basis of the time-varying values of the energy price). The aforementioned problem becomes very challenging to solve in a distributed way, as we shall see in the next section, due to the presence of coupling constraints. Namely, the power that the $i$-th MG sells to the $j$-th MG must obviously equal the power that the $j$-th MG buys from the $i$-th MG.

\section{2 | Problem formulation}

Following similar works ${ }^{25}$, we assume that the only state of a MG corresponds to the state of charge of its energy storage device (if more storage units are available, then we shall consider the aggregate set of all of them). Thus, we have that the overall state of the network of MGs, that aggregates all the states of the single MGs, is represented by

$$
x=\left[x_{1}, \ldots x_{M}\right]^{T} \in \mathbb{R}^{M}
$$

where $M$ is the number of microgrids. Then, the time-discrete dynamic equation of a MG is expressed by

$$
x_{i}(k+1)=x_{i}(k)+\beta_{c h, i} u_{c h, i}(k)-\beta_{d i s, i} u_{d i s, i}(k)
$$

where $x_{i}(k)$ is the state of charge of the storage device at time $k, \beta_{c h, i}$ and $\beta_{d i s, i}$ represent the charging/discharging efficiency coefficients for each storage device respectively, and $u_{c h, i}, u_{d i s, i} \geq 0$ are the charge and discharge powers, respectively. In addition, the problem is subject to a number of constraints as follows where over and under bar notations represent upper and lower bounds of relative variables, respectively:

$$
\underline{x}_{i} \leq x_{i}(k) \leq \bar{x}_{i} \quad\left\{\underline{x}_{i}, \bar{x}_{i}\right\} \in \mathbb{R}
$$




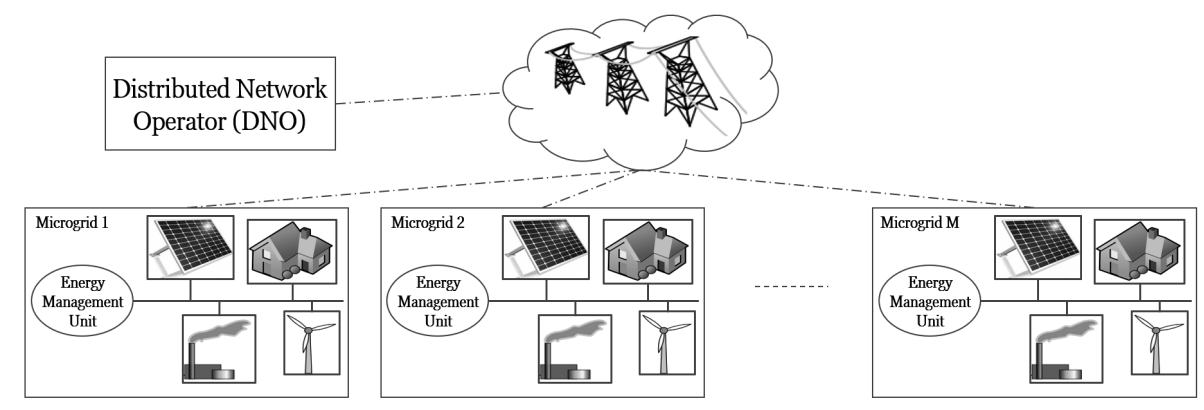

(a) The diagram depicts the topological scheme of the network. The dotted-dashed lines represent the communication from/to the MGS from/to the DNO.

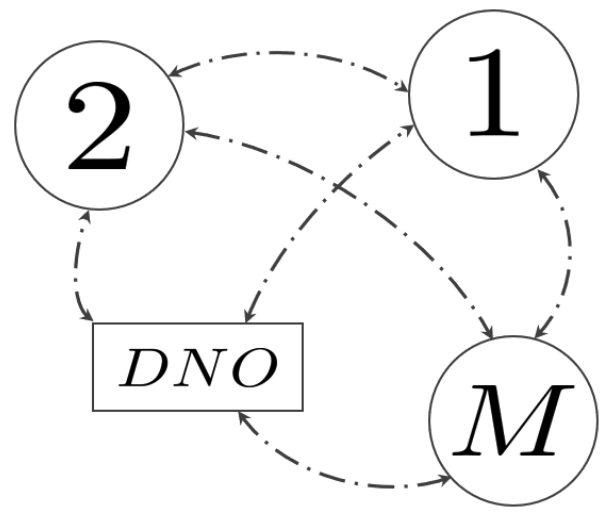

(b) The network of Microgrids and the Distributed Network Operator can be seen as a graph. Each MG, as well as the DNO, can be represented by a node of the graph. The edges represent the communication line.

FIGURE 1 Two different graphics to describe in an intuitive way the topology of the network of MGs and the Distributed Network Operator.

$$
\begin{gathered}
0 \leq u_{c h, i}(k) \quad \leq \bar{u}_{c h, i} \quad \bar{u}_{c h, i} \in \mathbb{R} \\
0 \leq u_{d i s, i}(k) \leq \bar{u}_{d i s, i} \quad \bar{u}_{d i s, i} \in \mathbb{R} \\
\Delta u_{b a l, i}(k)-\beta_{c h, i} u_{c h, i}(k)+\beta_{d i s, i} u_{d i s, i}(k)+u_{n e t, i}(k)+\sum_{j \in \mathcal{N}_{i}} u_{i j}(k)=0 . \\
\underline{u}_{n e t, i} \leq u_{n e t, i}(k) \leq \bar{u}_{n e t, i} \quad\left\{\underline{u}_{n e t, i}, \bar{u}_{n e t, i}\right\} \in \mathbb{R} \\
\underline{u}_{i j} \leq u_{i j}(k) \leq \bar{u}_{i j} \quad\left\{\underline{u}_{i j}, \bar{u}_{i j}\right\} \in \mathbb{R} \quad \forall j=1, \ldots, p_{i} \\
u_{n e t, i}(k) \leq 0 \quad \text { and } \quad u_{m g, i j}(k) \leq 0 \quad \text { if } \Delta u_{b a l, i}(k)>0 \\
u_{n e t, i}(k) \geq 0 \quad \text { and } \quad u_{m g, i j}(k) \geq 0 \quad \text { if } \Delta u_{b a l, i}(k)<0 . \\
u_{i j}(k)+u_{j i}(k)=0 \quad \forall j=1, \ldots, p_{i}
\end{gathered}
$$

Constraints (3-4) refer to the storage units. In particular, constraint (3) prevents one from overcharging or over-discharging the storage units. Constraint (4) takes into account that the charging and discharging rates in equation (2) are non-negative and upper bounded, according to the physics of the specific storage devices (e.g., batteries). Constraint (5) enforces the balancing constraint at the level of single MGs, where $\mathcal{N}_{i}$ is the set of the neighbors of the $i$-th MG, represented with the arcs of the network graph that involve the $i$-th MG. In particular, the (hourly) predicted power imbalance $\Delta u_{b a l, i}$ of the i-th MG (i.e., the difference between the generated and consumed power) needs to be covered by either charging/discharging the storage units, or by exchanging power with another MGs $j$ (denoted as $u_{i j}$ ) or with the DNO (denoted as $u_{n e t, i}$ ). Available hourly inaccurate predicted power imbalances have been considered in this work (similarly to other papers ${ }^{25}$ ), while it is assumed that the predictions are perfect when the horizon of prediction is equal to zero (i.e., there are no measurement errors). At this regard, constraint (6) is required to take into account the upper (positive, $\bar{u}_{n e t, i}$ ) and lower (negative, $\underline{u}_{n e t, i}$ ) bounds due to the power flow constraints in the power network. 
Here, negative values of $u_{n e t, i}$ correspond to sold energy, and positive values correspond to purchased energy. Similar power flow constraints are considered for the power exchange among MGs in the constraint (7). Here, non-negative values of $u_{i j}$ mean that power is purchased by the $i$-th MG from the $j$-th MG. Upper $\bar{u}_{i j}$ and lower $\underline{u}_{i j}$ bounds limit the amount of the exchanged power among MGs in (7). In case one MG can exchange power with only a subset of the other MGs, we use $p_{i}$ to denote the number of neighbors of the i-th MG. Finally, it is assumed that one MG can not simultaneously purchase and sell power to other MGs (similarly to other papers ${ }^{25}$ ) in constraints (8). In particular, one MG can sell power only if its predicted imbalance is positive (i.e., more power generated than consumed) otherwise it can only buy energy. Since the sign of the imbalances changes with time index, this needs to be taken explicitly into account. A further implicit equality constraint (namely, (9)) is required to enforce that the power purchased by the $\mathrm{j}$-th MG from the $\mathrm{i}$-th MG is equal to that sold by the $\mathrm{i}$-th MG to the $\mathrm{j}$-th one.

\section{3 | Objective Function and MPC formulation}

Assuming that the set of constraints listed in Section 2.2 is feasible, here we are interested in choosing the most convenient sequence of control actions of each MG to minimize the operational costs (possibly down to negative values that correspond to positive revenues for the MGs). As in the well-known spirit of MPC, at each time step a MG computes its optimal control sequence for the upcoming future horizon of time (using available non-exact predictions) and only implements the first control action $u_{i}^{*}(0)$ in the computed sequence, in terms of power exchanged. In particular, this is:

$$
u_{i}^{*}(0)=\left[u_{c h, i}^{*}, u_{d i s, i}^{*}, u_{n e t, i}^{*}, u_{i 1}^{*}, \ldots, u_{i p_{i}}^{*}\right]^{T} \in \mathbb{R}^{n_{i}+p_{i}}
$$

where $p_{i}$ is the number of neighbors of the $i$-th microgrid, $u_{n e t, i}^{*}$ is the (optimal) power exchanged with the distributed network operator, and $n_{i}=3$. The superscript * corresponds to an optimal computed value. As mentioned, $u_{n e t, i}$ and $\left[u_{i 1}, \ldots, u_{i p_{i}}\right]$ can assume both positive and negative values where, for our convention, positive values correspond to purchased power and negative values correspond to sold power. By collecting the input vector of each single MG we obtain the global input vector as

$$
u^{*}(0)=\left[u_{1}^{*}(0)^{T}, \ldots u_{M}^{*}(0)^{T}\right]^{T}
$$

We have to find an optimal control sequence $u_{i}^{*}, \forall i=1, \ldots, M$, along the prediction horizon $N$, given the current state $\hat{x}$, while respecting all the input and state constraints previously listed, (3)-(9), and here summarized as

$$
x(k) \in \mathbb{X}, \quad u(k) \in \mathbb{U} \quad k=0, \ldots, N-1
$$

where $\mathbb{X} \subset \mathbb{R}^{M}$ and $\mathbb{U} \subset \mathbb{R}^{\left(n_{i}+p_{i}\right) \cdot M}$ are compact polyhedral sets.

Given $\hat{x}$, and a finite-horizon input sequence $\mathbf{u}=(u(0), \ldots, u(N-1))$ we define the global cost function over the finite horizon as follows:

$$
\begin{aligned}
V(\hat{x}, \mathbf{u}) & =\sum_{k=0}^{N-1} \sum_{i=1}^{M} \ell_{i}(u(k)) \quad \text { s.t. } \\
x(0) & =\hat{x} \\
x(k+1) & =x(k)+B u(k) \quad k=0, \ldots, N-1
\end{aligned}
$$

in which $B=\operatorname{diag}\left[B_{1}, \ldots, B_{M}\right]$ is a block diagonal matrix with blocks $B_{i}=\left[\beta_{c h, i},-\beta_{d i s, i}\right]$ for $i=1, \ldots, M$ is accordingly defined from (2), and $\ell_{i}(\cdot)$ is the stage cost function, for each MG, given by:

$$
\ell_{i}(u)=F_{i} \cdot u_{i}=\left[0,0, c_{n e t, i}, c_{i 1}, \ldots, c_{i p_{i}}\right] \cdot\left[u_{c h, i}, u_{d i s, i}, u_{n e t, i}, u_{i 1}, \ldots, u_{i p_{i}}\right]^{T}
$$

where $\left[c_{i 1}, \ldots, c_{i p_{i}}\right]$ is a vector containing the prices of energy exchange between microgrid $i$ and its neighbors. We can now define the finite horizon optimal control problem (FHOCP) to be solved given the current state $\hat{x}$ as follows:

$$
\mathbb{P}(\hat{x}): \quad \min _{\mathbf{u}}\left\{V(\hat{x}, \mathbf{u}) \mid \mathbf{u} \in \mathcal{V}_{N}(\hat{x})\right\}
$$

where:

$$
V_{N}(\hat{x})=\{\mathbf{u} \mid u(k) \in \mathbb{U}, x(k) \in \mathbb{X}\}
$$

or equivalently, such that the set of the inequality and equality constraints (3)-(9) are fulfilled. Solving problem $\mathbb{P}(\hat{x})$ in (15) provides $\mathbf{u}^{*}(\hat{x})$ as the optimal input sequence, associated with a corresponding optimal state sequence $\mathbf{x}^{*}(\hat{x})=$ $\left\{x^{*}(0)=\hat{x}, x^{*}(1), \ldots, x^{*}(N)\right\}$. Then, as in the MPC framework, only the first element of the optimal input sequence is sent to 
the controlled system, i.e. $u=u^{*}(0)$. Finally, we recall that $\mathbb{P}(\hat{x})$ can be formulated as a Linear Programming Problem and effectively solved numerically.

\section{3 | THE PROPOSED DISTRIBUTED PREDICTIVE ENERGY MANAGEMENT}

\subsection{The decomposition framework}

One of the main challenges for solving these problems is the task of breaking the large scale system up into smaller ones and solving each one separately, either in parallel or sequentially. Decomposition in optimization is an old idea $28,29,30,31,32$ and several recent works described novel approaches to the decoupling strategy $33,34,35$. The decomposition task may be not trivial due to the fact that variables of each microgrids could be not separable. This implies that some of the decision variables can appear in more than one subproblems, i.e. $u_{i j}$ in (9).

This coupled structure can be represented by a hypergraph. Each nodes of the hypergraph is associated to a subproblem, which involves local variables, objective terms, and local constraints. Differently from classical edges, hyperedges can connect also more than two nodes. Each hyperedge can be associated to coupled variables or constraints among subproblems. Hence, this formulation allows to deal with the case of coupling constraints involving more than two subproblems. From the considered problem on microgrids, the private (local) variables are those that are associated only with the single microgrid and the public (interface) variables are those that are shared between two microgrids. Indeed in our case, the coupled constraints are those of constraint (9) and hence the hypergraph is actually a graph. However, the approach proposed in this section is valid for the more general case of hypergraphs.

Even if several methods can be used for decomposing the large scale system into smaller ones, we refer to the well established theory from ${ }^{36,37,38}$ in which two approaches are described: primal and dual decomposition. The former solves the original problem by solving the derived subproblems separately using an iterative method such as the subgradient method. The latter, instead, solves the original problem after introducing some new variables, and working with the dual problem. See ${ }^{37}$ for further details on the methods. In the next subsection the dual decomposition framework will be briefly recalled for reader convenience. Roughly speaking, from the graph standpoint, each subsystem has its own private copy of the public variables of the links it is adjacent to and an associated price. The subsystem uses this price to optimize its private and local copy of the public variables. The public variables on each link are then compared, and the prices are updated, in order to ensure consistency of the local copies of public variables (i.e. $u_{i j}$ ). Note that the goal is to obtain consistency without any exchange of private variables.

First, let us divide the optimization vector in (10) into private $w_{i}$ and public $y_{i}$ variables as:

$$
\begin{aligned}
w_{i} & =\left[u_{c h, i}, u_{d i s, i}, u_{\text {net }, i}\right]^{T} & & \in \mathbb{R}^{n_{i}} \\
y_{i} & =\left[y_{i j}\right]_{j \in \mathcal{N}_{i}} & & \in \mathbb{R}^{p_{i}} \\
u_{i} & =\left[w_{i}^{T}, y_{i}^{T}\right]^{T} & & \in \mathbb{R}^{n_{i}+p_{i}}
\end{aligned}
$$

where

$$
y_{i j}= \begin{cases}u_{i j} & \text { if } i<j \\ -u_{i j} & \text { if } i>j\end{cases}
$$

Thus, the inequality constraints described in (3) - (8) can be rewritten, in a compact form, as

$$
u_{i}=\left[w_{i}^{T}, y_{i}^{T}\right] \in \mathbb{U}_{i}(\hat{x}) \subseteq \mathbb{R}^{n_{i}+p_{i}} .
$$

Moreover, subsystems are coupled through constraints that require various subsets of the components of the public variables to be equal. This procedure can be described by collecting all the public variables together into a single vector $y=\left[y_{1}^{T}, \ldots, y_{M}^{T}\right]^{T} \in$ $\mathbb{R}^{p}$, where $p=p_{1}+\ldots+p_{M}$ is the total number of the public variables. We assume $K$ links exist, and we introduce a vector $z \in \mathbb{R}^{K}$ that gives the common values of the public variables on the links. We can express the coupling constraints as

$$
y=E z
$$

where $E \in \mathbb{R}^{p \times K}$ is defined by its elements:

$$
E_{l j}=\left\{\begin{array}{cc}
1 & y_{l} \in K_{j} \\
0 & \text { otherwise }
\end{array} \quad l=1, \ldots, p\right.
$$


where $y_{l}$ is to denote the $l$-th scalar component of $y$ for $l=1, \ldots, p$ and $K_{j}$ is the $j$-th link (i.e. the $j$-th link that constrains two local copies of a public variable to share the same value). The matrix $E$ specifies the list of the links, for the decomposition structure: it is the list that forces microgrids to share the local copies of the public variables. Let $E_{i} \in \mathbb{R}^{p_{i} \times K}$ denote the partitioning of the rows of $E$ into $M$ blocks associated with the different subsystems, so that $y_{i}=E_{i} z$. The matrix $E_{i}$ maps the vector of link variables into the public variables of subsystem $i$. Vector $z$ can be referred to as the vector of the link variables. Problem (15) then becomes,

$$
\mathbb{P}(\hat{x}): \quad \min _{\left\{\boldsymbol{w}_{i}, \boldsymbol{y}_{i}\right\}_{i=1}^{M}, \boldsymbol{z}}\left\{V\left(\hat{x}, \boldsymbol{w}_{i}, \boldsymbol{y}_{\boldsymbol{i}}\right)=\sum_{k=0}^{N-1} \sum_{i=1}^{M} \ell_{i}\left(w_{i}(k), y_{i}(k)\right)\right\}
$$

subject to (3) - (8), (13b),(13c) and

$$
y_{i}(k)=E_{i} z(k), \quad i=1, \ldots, M, k=0, \ldots, N-1
$$

where $\left(w_{i}(k), y_{i}(k)\right) \in \mathbb{R}^{n_{i}+p_{i}}$ for $i=1, \ldots, M, k=0, \ldots, N-1$, and

$$
\begin{aligned}
\boldsymbol{w}_{\boldsymbol{i}} & =\left(w_{i}(0)^{T}, w_{i}(1)^{T}, \ldots, w_{i}(N-1)^{T}\right)^{T} \\
\boldsymbol{y}_{\boldsymbol{i}} & =\left(y_{i}(0)^{T}, y_{i}(1)^{T}, \ldots, y_{i}(N-1)^{T}\right)^{T} \\
\boldsymbol{z} & =\left(z(0)^{T}, z(1)^{T}, \ldots, z(N-1)^{T}\right)^{T} .
\end{aligned}
$$

To better understand the framework it is useful to apply the previous formulation to an example of a network as depicted in Figure 2 .

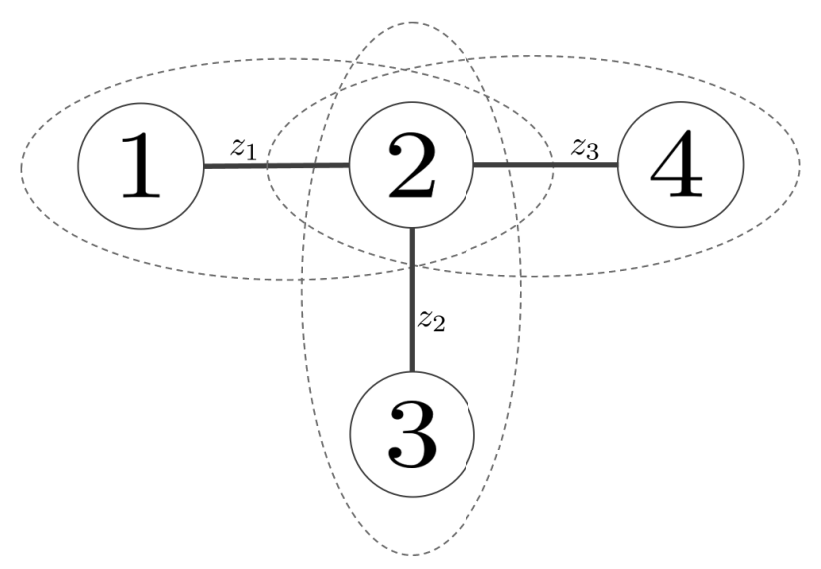

FIGURE 2 Example of a graph associated with 4 subproblems (nodes) with 3 shared variables (links): the vector of the common values of the public variables is $z=\left(z_{1}, z_{2}, z_{3}\right)$.

By following equation (21) we obtain

$$
E=\left[\begin{array}{c}
E_{1} \\
--- \\
E_{2} \\
--- \\
E_{3} \\
--- \\
E_{4}
\end{array}\right]=\left[\begin{array}{ccc}
1 & 0 & 0 \\
--- & --- & --- \\
1 & 0 & 0 \\
0 & 1 & 0 \\
0 & 0 & 1 \\
------ & --- \\
0 & 1 & 0 \\
--- & --- & --- \\
0 & 0 & 1
\end{array}\right] .
$$

Hence we have that $y_{1}=z_{1}, y_{2}=\left[z_{1}, z_{2}, z_{3}\right]^{T}, y_{3}=z_{2}, y_{4}=z_{3}$ with the meaning that subsystem 1 has a variable $\left(z_{1}\right)$ shared with subsystem 2 etc. 
With the proposed decomposition, the problem (22a) can be solved by each MG separately while the verification of the coupling constraint (22b) must be ensured.

\section{2 | Dual decomposition}

From (23), let us consider the following aggregate decision variables over the prediction horizon:

$$
\boldsymbol{w}=\left[\begin{array}{c}
\boldsymbol{w}_{1} \\
\boldsymbol{w}_{2} \\
\vdots \\
\boldsymbol{w}_{M}
\end{array}\right] \in \mathbb{R}^{n \times N}, \quad \boldsymbol{y}=\left[\begin{array}{c}
\boldsymbol{y}_{1} \\
\boldsymbol{y}_{2} \\
\vdots \\
\boldsymbol{y}_{M}
\end{array}\right] \in \mathbb{R}^{p \times N},
$$

with $n=\sum_{i=1}^{M} n_{i}$. By exploiting the dual decomposition structure, we can form the Lagrangian of problem (22a) subject to the constraints (22b) as:

$$
L(\boldsymbol{w}, \boldsymbol{y}, \boldsymbol{z}, v)=\sum_{i=1}^{M} \boldsymbol{\ell}_{i}\left(\boldsymbol{w}_{i}, \boldsymbol{y}_{\boldsymbol{i}}\right)+\operatorname{col}(v)^{T} \operatorname{col}(\boldsymbol{y}-E \boldsymbol{z})=\sum_{i=1}^{M}\left[\boldsymbol{\ell}_{i}\left(\boldsymbol{w}_{\boldsymbol{i}}, \boldsymbol{y}_{\boldsymbol{i}}\right)+\operatorname{col}\left(v_{i}\right)^{T} \operatorname{col}\left(\boldsymbol{y}_{\boldsymbol{i}}\right)\right]-\operatorname{col}(v)^{T} \operatorname{col}(E \boldsymbol{z})
$$

where $\boldsymbol{\ell}_{\boldsymbol{i}}$ is cost function of microgrid $i$-th along the prediction horizon $N, v \in \mathbb{R}^{p \times N}$ is the Lagrange multiplier (matrix) associated with $\boldsymbol{y}=\boldsymbol{E} \boldsymbol{z}, \operatorname{col}(\cdot)$ is the column-wise vectorization operator applied to a given matrix, and $v_{i} \in \mathbb{R}^{p_{i} \times N}$ is the submatrix of $v$ associated with the $i$-th subsystem. The minimization over $z$ results in the condition $E^{T} v=0$. This condition states that for each link, the sum of the Lagrange multipliers over the link is zero. By recalling the example in Figure 2 , we can obtain the set of feasible solutions as:

$$
E^{T} v=0 \Rightarrow v(k) \in<\left(\begin{array}{ccc}
1 & 0 & 0 \\
-1 & 0 & 0 \\
0 & 1 & 0 \\
0 & 0 & 1 \\
0 & -1 & 0 \\
0 & 0 & -1
\end{array}\right)>
$$

where $<A>$ denotes the span of matrix $A$ and $v(k)$ is the $k$-th column of $v$.

We now define the subproblem associated with the $i$-th subsystem:

$$
\begin{gathered}
\min _{\boldsymbol{w}_{i}, \boldsymbol{y}_{\boldsymbol{i}}} \boldsymbol{\ell}_{\boldsymbol{i}}\left(\boldsymbol{w}_{\boldsymbol{i}}, \boldsymbol{y}_{\boldsymbol{i}}\right)+\operatorname{col}\left(v_{i}\right)^{T} \operatorname{col}\left(\boldsymbol{y}_{\boldsymbol{i}}\right) \\
\text { subject to (3) - (8), (13b),(13c) }
\end{gathered}
$$

as a function of $v_{i}$. The dual of the original problem (22) is

$$
\begin{array}{cl}
\max _{\nu} & g(\nu)=\sum_{i=1}^{K} g_{i}\left(v_{i}\right) \\
\text { s.t. } \quad E^{T} v=0
\end{array}
$$

where $g_{i}(v)$ is the optimal value of the subproblem (28). We solve this dual decomposition master problem using a projected subgradient method. A subgradient for $-g_{i}$ at $v_{i}$ is simply $-\operatorname{col}\left(\boldsymbol{y}_{\boldsymbol{i}}\right)$, that represents the exchanged information among neighbors, i.e. the public variables as defined in Equation (9). However, following such a subgradient direction would not guarantee the condition $E^{T} v=0$. Then, the projection onto the feasible set $\left\{v \mid E^{T} v=0\right\}$ can be computed as

$$
\left[I-E\left(E^{T} E\right)^{-1} E^{T}\right] \boldsymbol{y}
$$

leading to the following update law for $v$ :

$$
\nu_{i}^{+}=v_{i}+\alpha\left(I-E\left(E^{T} E\right)^{-1} E^{T}\right) \boldsymbol{y}=v_{i}+\alpha(\boldsymbol{y}-E \hat{z})
$$

in which $\alpha$ is an appropriate step size (see Appendix A for details), and $\hat{z}$ represents an average of $\boldsymbol{y}$ over each link, given by:

$$
\hat{z}=\left(E^{T} E\right)^{-1} E^{T} y
$$

Note that each subproblem can be solved separately once the step $\alpha$ and the initial values of $v_{i}$ are available to all the MGs. In general, infeasible solutions may be locally obtained, due to the fact that each microgrid produces a local copy of the public variables related to its own constraints. Thus, if all primal subproblems (28) with $\boldsymbol{y}_{i}=E_{i} \hat{z}$ and $v_{i}$ fixed have a feasible solution, 
we obtain a feasible input sequence and an associated upper bound to the centralized problem (22). We finally note that the vector $v$ can be interpreted as the balancing prices for the supply demand market.

Let us summarize the distributed MPC formulation using the dual decomposition method. The subproblem solved by the $i$-th microgrid is:

$$
\mathbb{P}_{i}\left(\hat{x}_{i}\right): \quad \min _{\boldsymbol{w}_{i}, \boldsymbol{y}_{i}}\left\{V_{i}\left(\hat{x}_{i}, \boldsymbol{w}_{\boldsymbol{i}}, \boldsymbol{y}_{\boldsymbol{i}}\right)=\sum_{k=0}^{N-1}\left[\left[0,0, c_{n e t, i}(k)\right] \cdot w_{i}(k)+v_{i}^{T}(k) \cdot y_{i}(k)+\sum_{j \in \mathcal{N}_{i}} c_{i j}(k) \cdot y_{i}(k)\right]\right\}
$$

subject to $(3)-(8),(13 b),(13 c)$ and $(22 b)$.

Note that, at each optimization iteration, $v_{i}(k)$ is fixed. Our algorithm performs a further primal subproblem optimization using $\boldsymbol{y}_{i}=E_{i} \hat{z}$ to obtain a (possibly) feasible solution, and an associated upper bound on the optimal value for the minimization of the centralized problem:

$$
\mathbb{P}_{i}^{f}\left(\hat{x}_{i}\right): \quad \min _{\boldsymbol{w}_{i}}\left\{V_{i}^{f}\left(\hat{x}_{i}, \boldsymbol{w}_{i}, \boldsymbol{y}_{\boldsymbol{i}}\right)=\sum_{k=0}^{N-1}\left[0,0, c_{n e t, i}(k)\right] \cdot w_{i}(k)\right\}
$$

subject to (3) - (8), (13b) and (13c).

Finally, a pseudo-code algorithm can be formulated by considering the Model Predictive Control strategy together with the dual decomposition method as described in Algorithm 1.

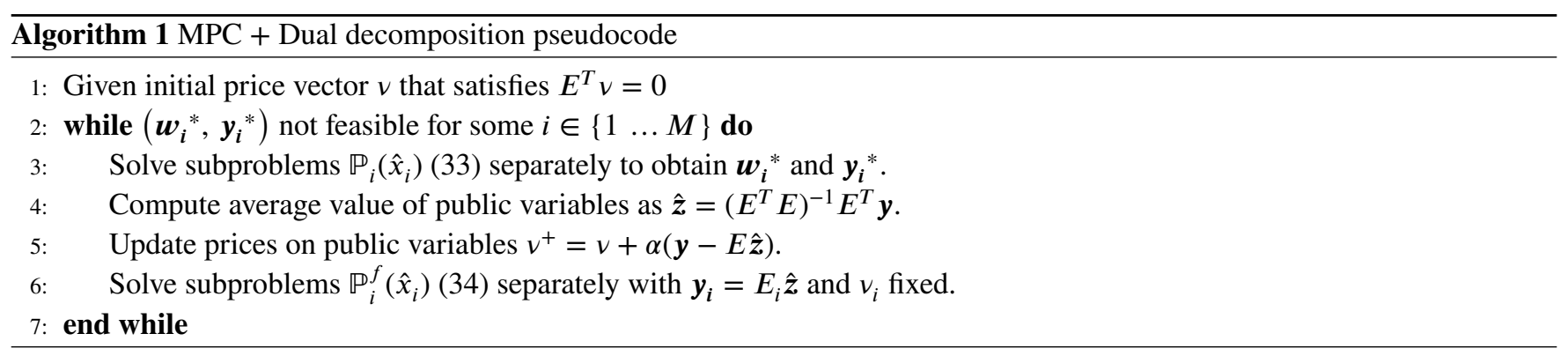

Note that communication takes place at Line 4. Moreover, iterations are performed until a feasible solution is found, i.e. a solution in which all public variables have reached a consensus and the power balance of all microgrids is respected, along with all other constraints. This implies that, in general, a sub-optimal solution is found. The level of sub-optimality can be evaluated by considering $\mathbb{P}_{i}\left(\hat{x}_{i}\right)$. By using the subgradient method in the dual problem for updating $v$, the smaller is the descent rate $(\boldsymbol{y}-E \hat{\boldsymbol{z}})$, the smaller is the distance of $\boldsymbol{w}_{\boldsymbol{i}}$ and $\boldsymbol{y}_{\boldsymbol{i}}$ from $\boldsymbol{w}_{\boldsymbol{i}}{ }^{*}$ and $\boldsymbol{y}_{\boldsymbol{i}}{ }^{*}$ respectively, in the primal problem. The dual decomposition theory states that the dual problem $\mathbb{P}_{i}^{f}\left(\hat{x}_{i}\right)$ is a lower bound with respect to primal problem $\mathbb{P}_{i}\left(\hat{x}_{i}\right)$. Due to the the convexity of the problem, when $\mathbb{P}_{i}^{f}\left(\hat{x}_{i}\right)$ and $\mathbb{P}_{i}\left(\hat{x}_{i}\right)$ have the same value, the optimality occurs. Hence, the smaller is the difference between primal and dual problem, the nearest is the control input sequence that provides the optimal solution of the primal problem.

\section{3 | A discussion on the distributed implementation approaches}

In the proposed method, each local controller decides how to fulfill the microgrid demand, by receiving or sending power towards the rest of the network in a distributed fashion. Usually, the problem of the optimal scheduling of the power flow in a network of MGs can be solved in a distributed way, by using an aggregator to gather and process the information arising from single MGs. In this way, the MGs do not have to exchange communications among themselves, while they only communicate to the aggregator. Then, the coupling constraints are solved in practice by the aggregator. From the standpoint of the communication network, this solution is decentralized, as MGs do not have to exchange information among themselves. Nonetheless, Algorithm 1 can be realized either by the adoption of an aggregator, or by exchanging peer-to-peer communications directly with the other nodes. This means our methodology could be implemented without using an aggregator as well. In this case, the MGs need to exchange information to find a consensus on the value of the coupled variables. From the standpoint of the communication network, this solution is distributed, as MGs need to exchange information among themselves. However, from the MPC perspective, if the 
overall system has an interacting dynamics among the subsystems is considered again as distributed. Then, in the distributed MPC literature there are two different subcategories in which the objective function is local or global, for the entire system. These approaches are called non-cooperative and cooperative, respectively. Further details are well described in literature ${ }^{11}$. Thus, for our standpoint a non-cooperative distributed MPC approach is considered and analyzed for the network of microgrids.

Essentially, the constraint (9) is the aim of the discussion and in the centralized formulation it is automatically included in the mathematical formalization. However, in a distributed way, it is not possible to handle it directly due to the fact that each microgrid has no information, at the beginning, about its neighbors. Thus, the presence or the absence of the aggregator distinguishes how this problem is tackled in a distributed approach. Figure 3 describes the two ways in which the algorithm can be implemented in practice. On the left, the two MGs do not exchange information directly, but an aggregator gathers the
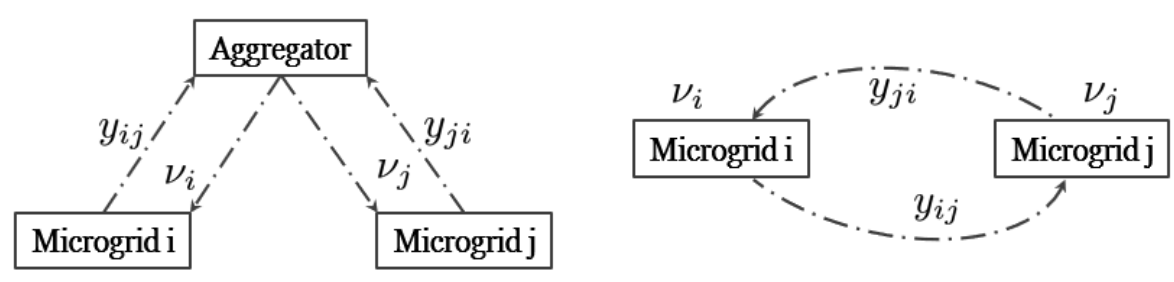

FIGURE 3 Different topologies the proposed algorithm is compliant with.

local copies of the public variables from the single MGs. On the right, the MGs directly exchange the values of the local copy of the public variable without needing any aggregator. In this case it is possible to implement a privacy-preservation strategy to avoid malicious (curious) attacks.

\section{4 | SIMULATION RESULTS AND DISCUSSION}

\section{1 | Case study description}

In order to assess the performance of the proposed approach, a network of five microgrids is considered. In this work, a complete graph is considered, thus $K=\sum_{i=1}^{M-1} i$. Let us recall that the stage cost function $\ell_{i}(\cdot)$ is defined as

$$
\ell_{i}(u)=F_{i} \cdot u_{i}=\left[0,0, c_{n e t, i}, c_{i j}\right] \cdot\left[u_{c h, i}, u_{d i s, i}, u_{n e t, i}, u_{i j}\right]^{T} \quad j=1, \ldots, p_{i} .
$$

The expected costs of purchasing/selling power from/to the distribution network operator and the other microgrids $\left(c_{n e t, i}, c_{i j}\right.$ respectively) are modeled as constant values equal to $0.08 \$ / \mathrm{kWh}$ between 0 and 6 A.M. and represented by independent distributed random vectors ranging between 0.075 and $0.14 \$ / \mathrm{kWh}$ for the rest of the day ${ }^{25}$. Figure 4 shows a realization of the costs that is used in the simulation. In this case we are using a day-ahead prices during the prediction horizon $N$. This is again consistent with the choice of ${ }^{25}$. At the next time step, then the price vector is shifted accordingly.

Each microgrid is equipped with two different renewable generators (a wind turbine and a photovoltaic module), uncontrollable loads, and an energy storage system. These components have certain bounds as specified in Equations (3) - (8). The maximum capacity of the energy storage system is between $5\left(\underline{x}_{i}\right)$ and $500\left(\bar{x}_{i}\right) \mathrm{kWh}$ for all microgrids. The maximum charge and discharge powers are all equal, and limited to $50 \%$ of the energy storage system capacity per hour $\left(\bar{u}_{c h, i}=\bar{u}_{d i s, i}=250 \mathrm{~kW}\right)$. The power exchanged with the DNO must be less than $100 \mathrm{~kW}$ in absolute value (i.e. purchase or sold). The maximum power that microgrids can exchange with each other is $125 \mathrm{~kW}$ (in absolute value). Initial charge of the energy storage system is $\hat{x}_{0, i}=5$ $\mathrm{kWh}$ for each micro-grid $i=1, \ldots, M$. This is consistent with the assumption of previous works ${ }^{25}$.

$$
\begin{gathered}
5 \leq x_{i} \leq 500 \quad(\mathrm{kWh}) \\
0 \leq u_{c h, i} \leq 250 \quad(\mathrm{~kW}) \\
0 \leq u_{d i s, i} \leq 250 \quad(\mathrm{~kW}) \\
-100 \leq u_{n e t, i} \leq 100 \quad(\mathrm{~kW}) \\
-125 \leq u_{i j} \leq 125 \quad(\mathrm{~kW})
\end{gathered}
$$



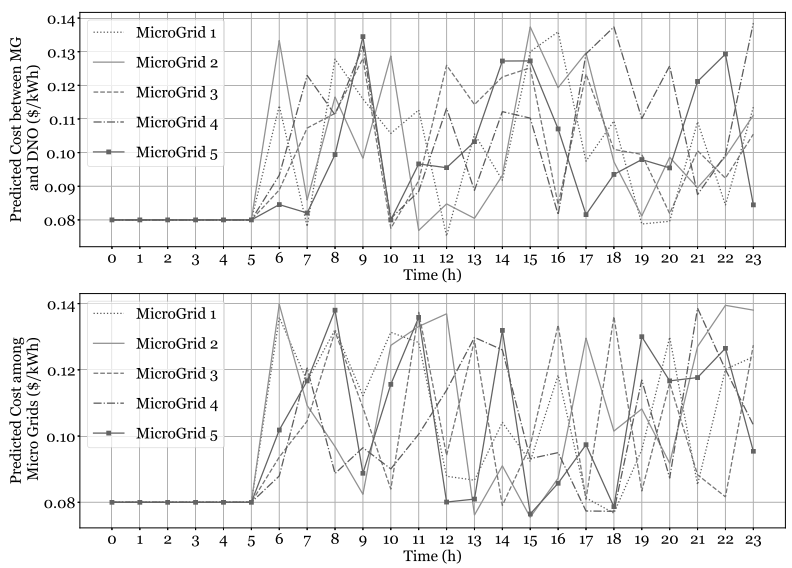

FIGURE 4 Exchange costs among microgrids and the distributed network operator.

TABLE 1 Initial predicted power balance $\left(\Delta u_{b a l, i}\right)(\mathrm{kW})$.

\begin{tabular}{lccccc}
\hline Hour & Microgrid 1 & Microgrid 2 & Microgrid 3 & Microgrid 4 & Microgrid 5 \\
\hline 1 & 9.67079 & -37.4347 & -26.494 & -18.728 & -10.3645 \\
2 & 39.0944 & 3.4149 & 43.3593 & 47.7657 & 12.9227 \\
3 & -8.97945 & -42.5555 & -38.1107 & 36.6328 & -19.5104 \\
4 & 5.48782 & 37.3142 & 17.6692 & 45.6643 & -18.2151 \\
5 & 44.8317 & 36.6844 & 38.9273 & -36.6203 & 28.1923 \\
6 & 28.3048 & 40.8821 & -31.5469 & -43.7577 & -46.7124 \\
7 & -3.97557 & -13.8361 & 9.76554 & -44.3611 & -42.5498 \\
8 & -3.58206 & 4.32963 & -25.2645 & -0.715182 & -32.436 \\
9 & -40.536 & -49.1489 & -30.788 & 30.8216 & 24.6507 \\
10 & 13.9433 & 25.1515 & -38.7525 & -48.1786 & 45.7398 \\
11 & -20.8858 & 34.7042 & 47.0619 & -32.6539 & -0.94774 \\
12 & -23.3351 & -36.1138 & 40.2481 & 23.408 & -11.1987 \\
13 & -2.96255 & -23.5074 & -42.2645 & 48.3192 & 19.6489 \\
14 & -15.8886 & 0.709367 & 4.03815 & -19.0965 & 5.99215 \\
15 & 16.9505 & -43.5514 & 47.6784 & 37.2654 & 10.535 \\
16 & 0.140528 & -46.4334 & 11.9735 & -21.9273 & 3.46699 \\
17 & 28.8477 & 2.41981 & 34.4541 & 37.8464 & -4.10521 \\
18 & 11.9132 & -44.6631 & -29.0382 & -13.326 & -32.2797 \\
19 & -48.36 & -37.1291 & -45.2477 & -10.0116 & 46.8043 \\
20 & 34.5849 & -6.84211 & 8.35187 & 15.7509 & 44.3326 \\
21 & 27.7089 & 7.46616 & -37.2975 & -14.7768 & 1.72214 \\
22 & 13.5405 & 40.2782 & -22.6432 & -20.6672 & -36.0828 \\
23 & -42.1615 & -27.3988 & 30.8939 & 24.8035 & -33.5008 \\
24 & -20.1591 & 48.7584 & 13.7667 & 42.4103 & 29.3526 \\
\hline
\end{tabular}

Only the hourly estimated power balances are required to solve the optimization problem ${ }^{25}$. That is, the difference between generated and consumed energy. Then, in order to take into account the fact that predictions cannot be perfect, we add some artificial noise (as $\pm 10 \%$ of the nominal value) to test the robustness of the proposed method. In the following we set the prediction horizon equal to $N=12$. The initially predicted power balances are show in Table 1 . 
In the MPC strategy, at each time instant we apply only the first of the optimal input sequence $\mathbf{u}_{i}^{0}$. Thus, at the next time instant new predictions for the power balances are required. This can be done in practice by using a forecasting technique to predict the uncertain variables. With such a short future horizon of interest (i.e. 12 hours) predictions are usually quite accurate.

Finally, a diminishing step-size for the projected subgradient method in the MPC using dual decomposition algorithm is used, as detailed in Appendix A. In these simulation scenarios we set:

$$
\alpha_{k}=\frac{1}{\sqrt{1+20 \cdot c}}
$$

where $c$ is the current number of the cooperative iteration.

\section{2 | Scenario 1: Centralized vs. Distributed approach}

Figure 5 shows the comparison between the instantaneous optimal costs of the distributed algorithm with respect to the centralized one. The centralized version (orange continuous line) produces similar results with respect to those depicted in literature ${ }^{25}$

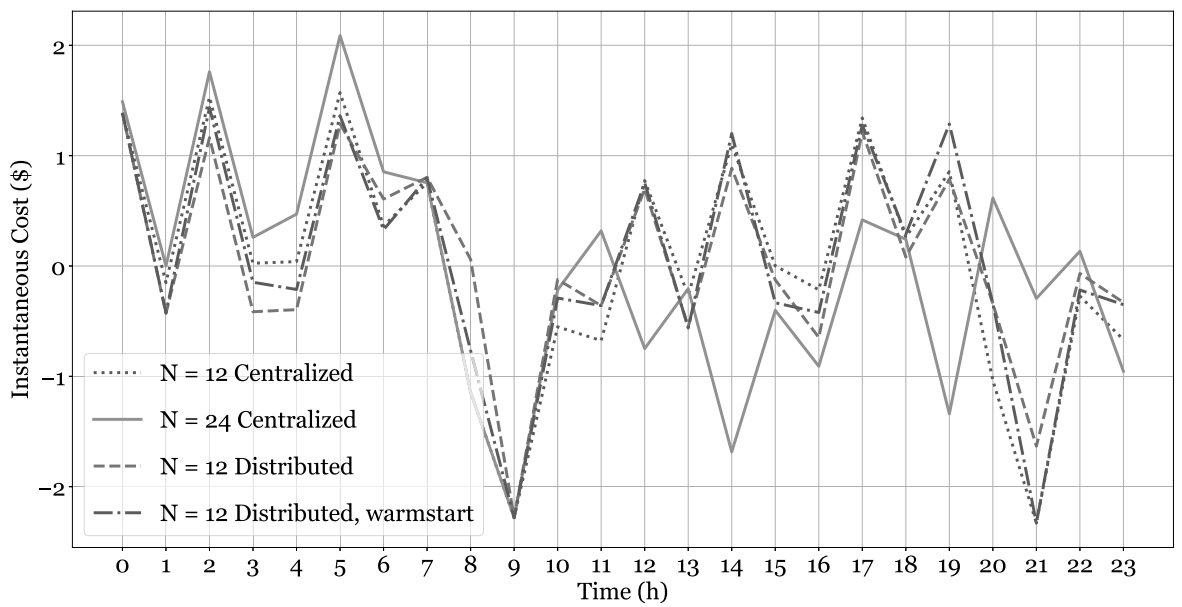

FIGURE 5 Comparison between instantaneous centralized and distributed computed optimal costs with a prediction horizon length set equal to 12 hours.

for the same case study (with very minor differences caused by stochastic fluctuations of prices). A centralized 12 hour prediction horizon version is also presented (the blue dotted line). However, the proposed distributed approach (the green dashed line) provides comparable results with the centralized version, especially when the prediction horizon is the same (12 hours). A suboptimal and feasible solution, i.e. a consensus, is always achieved within a certain number of cooperative iterations. Although coupled variables represent the hard constraints in terms of MPC formulation, the use of the iterative algorithm of the dual decomposition method allows the receding horizon control problem to find a distributed feasible, and sub-optimal, solution of the centralized (hardly scalable) problem. Even if distributed solutions are half of the time better than the centralized solution, the centralized approach knows all about the constraints and the state of charge of the energy storage units of each MG. Thus, it generates the optimal revenue in the considered time interval, by taking into account all the prediction horizon. This fact will be clear in Figure 6 in which the cumulative cost is presented. However, note that suboptimal methodologies, such as the proposed one, may still occasionally outperform the optimal solution in some sub-intervals of the considered time interval. In the warm-start case, at the end of each decision time, the columns of $v$ previously calculated are shifted 1 hour forward, adding a zero column at the end, and are assigned again as a warm start of the algorithm, rather than being set to 0 as in the standard distributed approach.

Figure 6 shows the aggregated costs of each implemented algorithm to evaluate the long term advantages of the proposed method. The aggregated cost is obtained by integrating each cost hour-by-hour. Thus, it is quite intuitive to see that the centralized version with 24 hours as prediction horizon produces the greater revenue in a long term scenario (see the aggregated cost at time 23). However, also the 12 hour prediction horizon version produces similar results. The distributed algorithms (with or without 


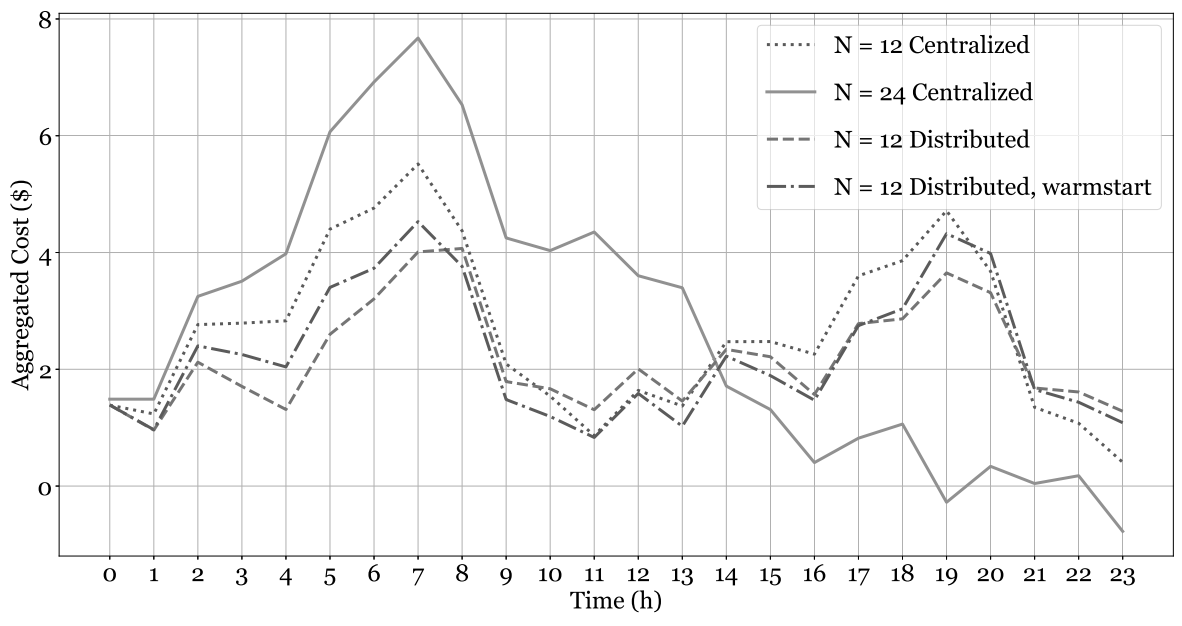

FIGURE 6 Comparison between aggregated centralized and distributed computed optimal costs with a prediction horizon length set equal to 12 hours.

a warm-start feature) produce in the long a lower revenue. Even if they are similar during the overall simulation, the warm-start produce a slight improvement in the final result, i.e., the global revenue of the network. Given the linear nature of the model and the convexity of the optimization problem it is possible to note how the warm-start solution produces a slightly better revenue with respect to the non-warm-start approach, as expected.

Notice that the centralized version can be considered, in the selected time interval, as a lower bound in terms of cost (or an upper bound in terms of revenues) for the purchased/sold energy. Also note that solutions obtained with different prediction horizons and different time intervals generate different, and in principle non-comparable, solutions.

\section{3 | Scenario 2: Open-loop vs. Closed-loop}

Figure 7 shows the comparison between an Open-loop and a Closed-loop implementation, in the case where some variations of the power balances reported in Table 1 occur. These variations are unknown to the controllers. The Open-loop approach, at

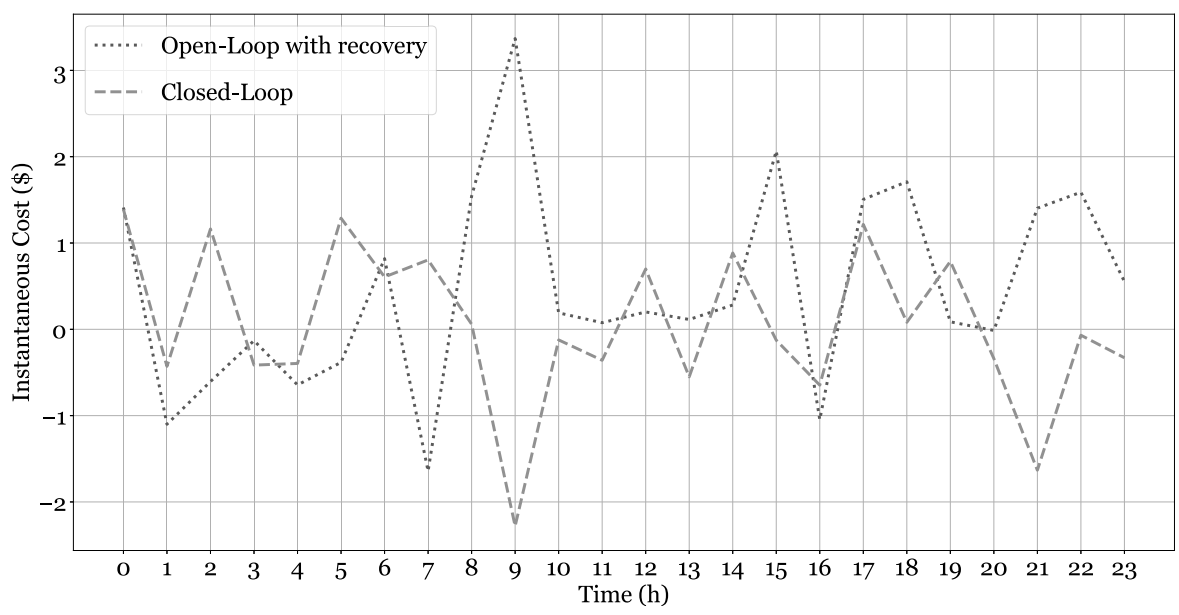

FIGURE 7 Comparison between instantaneous cost of Open-loop and Closed-loop implementations; the dot-dashed line represents the Open-loop strategy with a recovery strategy; the dashed line is the previously mentioned Closed-loop strategy with a prediction horizon equal to 12 . 
the first time instant, provides a 24 hour prediction horizon solution. The overall optimal input control sequence is then applied for all the following 24 hours ${ }^{25}$. However, due to the gap between estimated and measured power balance, a recovery strategy is necessary to prevent infeasibility issues. To this aim, two different scenarios are possible:

1. If the generated power is greater than the expected one, the microgrid cannot sell to the distributed network operator or to the other microgrids. Thus, if the state of charge of the battery is lower than the upper bound, each MG loads the energy storage system device. If it is not possible, the power in excess is discarded.

2. If the generated power is lower than the expected one, the MGs can draw energy for their storage unit, and if this is not enough to balance or possible, because the battery state-of-charge is at the lower bound, they are allowed to purchase some extra power from the grid.

The Closed-loop solution, instead, implements a receding horizon control approach (only the first control action is applied). Then, a new measurement (of the actual power balance) is obtained and a new control problem is solved. This makes the problem always feasible with respect to small power balance variations as expected.

Figure 8 shows the comparison between the Open-loop and the Closed-loop strategies when the cost is aggregated along the time intervals, i.e., it is integrated to provide a long term cost analysis. Although the Open-loop solution seems to have a greater

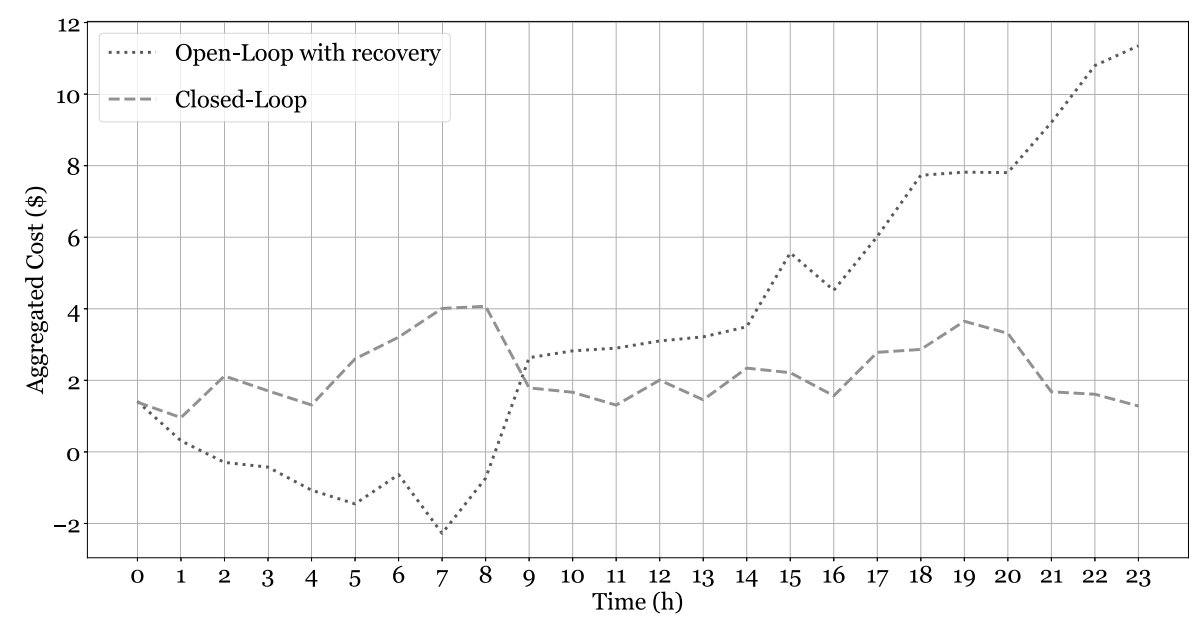

FIGURE 8 Comparison between the aggregated cost of the Open-loop strategy with respect to the Closed-loop implementations.

revenue in the first time intervals, due to an exploitation of the storage system, the aggregated cost, as shown in Figure 8 , demonstrates that it is not true in the long term. Since, the Open-loop approach does not consider the gap between the estimated and measured power balances, this leads, in the specified time interval, the non-achievement of the cooperative global objective of minimizing the overall net costs.

On the other hand, the receding horizon control approach leads to a greater revenue, given that it takes into account the latest measurement of the actual power balance, recomputing at each decision time the new optimal control sequence. Thus, the aggregated cost analysis further demonstrates how a Closed-loop strategy can be parsimonious with respect to an Open-loop strategy.

Even if the focus of the presented results was on a closed-loop solution, the goal of Figure 8 was to remark that a predictive closed-loop strategy is essential in this type of scenario in which each single MG has no knowledge about the neighbors state and the constraints. In fact, although in the first part of the time interval the open-loop strategy (with recovery) seems to have greater revenue, it presents a significantly higher aggregate cost at the end of the day. 


\section{5 | CONCLUSIONS AND FUTURE WORKS}

This paper presented a cooperative distributed approach for an optimal predictive power scheduling in a network of microgrids. The proposed methodology is based on a dual-decomposition algorithm, and it allows each microgrid to optimize, by solving linear programs independently, private power variables (exchange with DNO and charging/discharging of batteries) and public power variables (exchange among microgrids) also taking into account state-of-charge constraints. Iterations on dual variables are performed so that the microgrids cooperate towards the centralized optimum. Such an update occurs due to communication of the public variables, either to an aggregator or directly among microgrids. One remarkable feature of the proposed distributed methodology is that each microgrid does not need to share private variables. The algorithm is implemented in closed-loop according to the standard receding horizon principle, common to MPC algorithms. That is, each microgrid solves an optimization problem in which variables are optimized over a prediction horizon of 12 hours, but then only the optimal power variables of the first hour are actually implemented, and the overall optimization problems are solved again at the next hour.

The simulation results depict that a consensus is always reached and a slightly sub-optimal solution with respect to a centralized version is found. The mathematical formalization shows that the algorithm is completely scalable and it can be applied to a large scale system. Simulation of operational scenarios were presented in order to validate numerically the algorithm performances. In particular, results show how the distributed MPC approach allows one to cope effectively with differences between power balance predictions and actual values, e.g. due to different generation from renewable sources and different power consumption from loads. It is worth pointing out that the proposed dual decomposition based distributed MPC algorithm is able to cope with the difficulties associated with the coupling constraints of power exchanges among the microgrids.

Future works can concern a mathematical formalization of an extension of the presented algorithm in order to handle a timevarying behavior of the microgrids. This deals with the possibility that a single microgrid could go offline for a while and then become operating again. Another opportunity is to expand the microgrid dynamics, from an electrical standpoint, to test the predictive distributed algorithm with a de-facto standard power systems analysis and simulation software ${ }^{39}$.

\section{References}

1. C Dou, M Lv, T Zhao, Y Ji, H Li. Decentralised coordinated control of microgrid based on multi-agent system. IEEE Generation, Transmission and Distribution. 2015;9(16):2474-2484.

2. A Nisar, MS Thomas. Comprehensive Control for Microgrid Autonomous Operation With Demand Response. IEEE Transactions on Smart Grid. 2016;8(5):2081-2089.

3. P Vorobev, PH Huang, M Al Hosani, JL Kirtley, K Turitsyn. A Framework for Development of Universal Rules for Microgrids Stability and Control. 56th Annual Conference on Decision and Control (CDC), Melbourne, Australia. $2017 ;: 5125-5130$.

4. E Dall'Anese, H Zhu, GB Giannakis. Distributed optimal power flow for smart microgrids. IEEE Transactions on Smart Grid. 2013;4(3):1464-1475.

5. GW Arnold. Challenges and opportunities in smart grid: A position article. Proceedings of the IEEE. 2011;99(6):922-927.

6. P Lombardi, M Powalko, K Rudion. Optimal operation of a virtual power plant. Power \& Energy Society General Meeting, 2009. PES'09. IEEE. 2009;:1-6.

7. D Aloini, E Crisostomi, Raugi M Rizzo R. Optimal Power Scheduling in a Virtual Power Plant. Innovative Smart Grid Technologies (ISGT) Europe. 2011;.

8. P Ferraro, E Crisostomi, R Shorten, F Milano. Stochastic frequency control of grid-connected microgrids. IEEE Transactions on Power Systems. 2018;33(5):5704-5713.

9. H Dagdougui, R Minciardi, A Ouammi, M Robba, R Sacile. A dynamic decision model for the real-time control of hybrid renewable energy production systems. IEEE Systems Journal. 2010;4(3):323-333.

10. JM Maciejowski. Predictive control: with constraints. Pearson education; 2002. 
11. JB Rawlings, DQ Mayne. Model predictive control: Theory and design. Nob Hill Pub. Madison, Wisconsin; 2009.

12. G Pannocchia, JB Rawlings, SJ Wright. Conditions under which suboptimal nonlinear MPC is inherently robust. Systems \& Control Letters. 2011;60(9):747-755.

13. Le Xie, MD Ilic. Model predictive economic/environmental dispatch of power systems with intermittent resources. Power \& Energy Society General Meeting, 2009. PES'09. IEEE. 2009;:1-6.

14. A Parisio, L Glielmo. Energy efficient microgrid management using model predictive control. Decision and Control and European Control Conference (CDC-ECC), 2011 50th IEEE Conference on. 2011;:5449-5454.

15. A Parisio, C Wiezorek, T Kyntäjä, J Elo, KH Johansson. An MPC-based energy management system for multiple residential microgrids. Automation Science and Engineering (CASE), 2015 IEEE International Conference on. 2015;:7-14.

16. P Mc Namara, RR Negenborn, B De Schutter, G Lightbody. Weight optimisation for iterative distributed model predictive control applied to power networks. Engineering Applications of Artificial Intelligence. 2013;26(1):532-543.

17. A Ferramosca, D Limón, I Alvarado, EF Camacho. Cooperative distributed MPC for tracking. Automatica. 2013;49(4):906914.

18. M Razzanelli, G Pannocchia. Parsimonious cooperative distributed MPC algorithms for offset-free tracking. Journal of Process Control. 2017;60:1-13.

19. Y Zhang, N Gatsis, GB Giannakis. Robust energy management for microgrids with high-penetration renewables. IEEE Transactions on Sustainable Energy. 2013;4(4):944-953.

20. MS Rahman, AMT Oo. Distributed multi-agent based coordinated power management and control strategy for microgrids with distributed energy resources. Energy Conversion and Management. 2017;139:20-32.

21. Barreiro-Gómez Julián, Ocampo-Martinez Carlos, Quijano Nicanor. On the communication discussion of two distributed population-game approaches for optimization purposes. IFAC-PapersOnLine. 2017;50(1):11782-11787.

22. A Parisio, C Wiezorek, T Kyntäjä, Elo J Johansson KH. Cooperative MPC-based energy management for networked Microgrids. IEEE Transactions on Smart Grid. 2017;8(6):3066-3074.

23. BT Stewart, AN Venkat, JB Rawlings, SJ Wright, G Pannocchia. Cooperative distributed model predictive control. Systems \& Control Letters. 2010;59(8):460-469.

24. R Halvgaard, L Vandenberghe, NK Poulsen, H Madsen, JB Jørgensen. Distributed model predictive control for smart energy systems. IEEE Transactions on Smart Grid. 2016;7(3):1675-1682.

25. A Ouammi, H Dagdougui, L Dessaint, R Sacile. Coordinated model predictive-based power flows control in a cooperative network of smart microgrids. IEEE Transactions on Smart grid. 2015;6(5):2233-2244.

26. Ananduta Wicak, Barreiro-Gomez Julian, Ocampo-Martinez Carlos, Quijano Nicanor. Resilient Information-Exchange Protocol for Distributed Model Predictive Control Schemes. In: :1286-1291IEEE; 2018.

27. M Cao, AS Morse, BDO Anderson. Reaching a consensus in a dynamically changing environment: A graphical approach. SIAM Journal on Control and Optimization. 2008;47(2):575-600.

28. GB Dantzig, P Wolfe. Decomposition principle for linear programs. Operations research. 1960;8(1):101-111.

29. DP Bertsekas. Nonlinear programming. Athena scientific Belmont; 1999.

30. FP Kelly, AK Maulloo, DKH Tan. Rate control for communication networks: shadow prices, proportional fairness and stability. Journal of the Operational Research society. 1998;49(3):237-252.

31. L Xiao, M Johansson, S Boyd. Simultaneous routing and resource allocation via dual decomposition. IEEE Transactions on Communications. 2004;52(7):1136-1144. 
32. M Chiang, SH Low, AR Calderbank, JC Doyle. Layering as optimization decomposition: A mathematical theory of network architectures. Proceedings of the IEEE. 2007;95(1):255-312.

33. Tang Wentao, Daoutidis Prodromos. Network decomposition for distributed control through community detection in inputoutput bipartite graphs. Journal of Process Control. 2018;64:7-14.

34. Barreiro-Gomez Julian. Distributed Predictive Control Using Density-Dependent Population Games. In: Springer 2019 (pp. 111-132).

35. Barreiro-Gomez Julian. The role of population games in the design of optimization-based controllers: a large-scale insight. Springer; 2018.

36. S Boyd, L Xiao, A Mutapcic. Subgradient methods. lecture notes of EE392o, Stanford University, Autumn Quarter. 2003;2004:2004-2005.

37. S Boyd, L Xiao, A Mutapcic, J Mattingley. Notes on decomposition methods. Notes for EE364B, Stanford University. 2007;:1-36.

38. S Boyd, N Parikh, E Chu, B Peleato, J Eckstein, others . Distributed optimization and statistical learning via the alternating

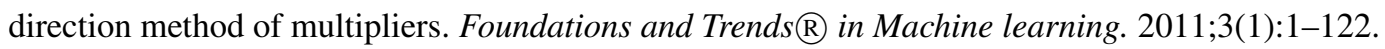

39. F Milano. A Python-based software tool for power system analysis. Power and Energy Society General Meeting (PES), 2013 IEEE. 2013;:1-5.

40. NZ Shor. Minimization methods for non-differentiable functions. Springer Science \& Business Media; 2012.

41. NZ Shor. Nondifferentiable optimization and polynomial problems. Springer Science \& Business Media; 2013.

42. BT Polyak. Introduction to Optimization, Optimization Software, Inc. Publ. Division. 1987.

\section{APPENDIX}

\section{A DIMINISHING STEP-SIZE}

The choice of the step length in subgradient methods is different from the case of standard gradient methods, and we refer to the literature ${ }^{40,41,42}$ for a detailed discussion on projected subgradient methods. It can be shown that for constant step size and constant step length, the (projected) subgradient algorithm is guaranteed to converge within some range of the optimal value ${ }^{36}$. Instead, for the diminishing step size and step length rules the algorithm is guaranteed to converge to the optimal value. In our case the nonsummable diminishing strategy was involved. The step size satisfies

$$
\alpha_{c} \geq 0, \quad \lim _{c \rightarrow \infty} \alpha_{c}=0, \quad \sum_{c=1}^{\infty} \alpha_{c}=\infty
$$

Step sizes that satisfy these conditions are called diminishing step size rules. In this paper we set $\alpha_{c}$ as:

$$
\alpha_{c}=\frac{a}{\sqrt{1+b \cdot c}}
$$

where $a, b>0$, and $c$ is the index of the current iteration of the algorithm. 